LBL-35997

UC-414

\title{
Crab Crossing in a Gamma-Gamma Collider
}

\author{
Ming Xie, Kwang-Je Kim, and Andrew Sessler \\ Accelerator and Fusion Research Division \\ Lawrence Berkeley Laboratory \\ University of California \\ Berkeley, California 94720
}

August 1994

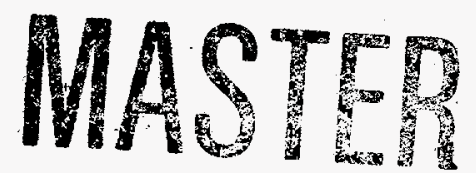

This work was supported by the Director, Division of Nuclear and High Energy Physics, of the U.S. Department of Energy under Contract No. DE-AC03-76SF00098. 


\section{DISCLAIMER}

This report was prepared as an account of work sponsored by an agency of the United States Government. Neither the United States Government nor any agency thereof, nor any of their employees, make any warranty, express or implied, or assumes any legal liability or responsibility for the accuracy, completeness, or usefulness of any information, apparatus, product, or process disclosed, or represents that its use would not infringe privately owned rights. Reference herein to any specific commercial product, process, or service by trade name, trademark, manufacturer, or otherwise does not necessarily constitute or imply its endorsement, recommendation, or favoring by the United States Government or any agency thereof. The views and opinions of authors expressed herein do not necessarily state or reflect those of the United States Government or any agency thereof. 


\section{DISCLAIMER}

Portions of this document may be illegible in electronic image products. Images are produced from the best available original document. 
LBL-35997

CBP Note-97

\title{
Crab Crossing in a Gamma-Gamma Collider*
}

\author{
Ming Xie, Kwang-Je Kim and Andrew Sessler \\ Center for Beam Physics \\ Lawrence Berkeley Laboratory \\ University of California \\ Berkeley, CA 94720
}

August, 1994

\begin{abstract}
The crabbing of an incident photon beam from a laser, and the electron beam with which it interacts at the conversion point, is shown to have the same efficiency as in head-on Compton scattering, but with the advantages of a crossing geometry. The resulting $\gamma$-ray beam is also crabbed, which allows for a crossing collision point, while maintaining the luminosity at the same value it would have in a head-on collision.
\end{abstract}

Submitted to the Proceedings of Gamma-Gamma Collider Workshop to be published in Nuclear Instruments and Methods in Physics Research A

* This work was performed under the auspices of the U.S. Department of Energy, Division of Nuclear and High Energy Physics, under contract No. DE-AC03-SF-00098 at the Lawrence Berkeley Laboratory. 


\section{Introduction}

The crabbing of electron and photon beams allows the conversion efficiency of photons into $\gamma$-rays to remain as high as it would be in head-on Compton scattering. In order to achieve this it is required to crab both the photon and electron beams. These aspects of the scheme are addressed in the next two sections of this paper.

If the electron beam is crabbed the resulting $\gamma$-ray beam is crabbed, without regard to the incident photon beam. Thus it is possible to have the crossing of $\gamma$-rays at an angle. This is highly advantageous for the collider in two regards. Firstly, the $\gamma$-ray crossing allows the debris of the crossing to go to one side. This is the same advantage as in $\mathrm{e}^{+}-\mathrm{e}^{-}$ colliders. The second advantage, special to $\gamma-\gamma$ colliders, is that there need be no holes made in the laser mirrors either for the opposing beam or for the collision debris, in order to arrange for a head-on Compton scattering.

A schematic, showing the layout for the proposed configuration, is presented in Fig.(1). The basic idea, that of "crabbing" of an electron beam was due to Robert Palmer [1], introduced first for linear colliders, and subsequently taken over for storage rings [2]. It has been noted that a similar approach can be applied to the $\gamma-\gamma$ collision [3]. In this paper, we extend the idea of crabbing further to photon beams and provide some relevant formulae.

\section{Crabbing of the Electron Beam}

The luminosity for two unequal bunches of Gaussian shape colliding with a crossing angle $2 \theta$ is (see Fig.(2))

$$
L(\theta)=\frac{N_{1} N_{2} f}{2 \pi \sqrt{\left(\sigma_{y 1}^{2}+\sigma_{y 2}^{2}\right)\left[\left(\sigma_{z 1}^{2}+\sigma_{z 2}^{2}\right) \theta^{2}+\left(\sigma_{x 1}^{2}+\sigma_{x 2}^{2}\right)\right]}} .
$$

Here $f$ is the collision frequency, $N_{i}$ is the number of the particle in the bunch $i$, and $\sigma_{x i}$, $\sigma_{\mathrm{yi}}$ and $\sigma_{\mathrm{zi}}$ are the rms bunch dimensions of the bunch $\mathrm{i}$ in the $\mathrm{x}-, \mathrm{y}$ - and $\mathrm{z}$-direction respectively. We assume that the bunches are not crabbed, i.e. the bunch axis lies in the direction of motion, that the collision is taking place in the $x-z$ plane, that all particles within each bunch are parallel to each other and that the bunch velocity in the $\mathrm{x}$-direction is non-relativistic, i.e., $\theta \ll 1$.

From Eq.(1), we obtain the reduction due to the crossing angle as follows: 


$$
\frac{\mathrm{L}(\theta)}{\mathrm{L}(0)}=\frac{1}{\sqrt{1+\left(\theta / \theta_{0}\right)^{2}}}
$$

where

$$
\theta_{0}=\sqrt{\frac{\sigma_{\mathrm{x} 1}^{2}+\sigma_{\mathrm{x} 2}^{2}}{\sigma_{\mathrm{z} 1}^{2}+\sigma_{\mathrm{z} 2}^{2}}}
$$

In order to avoid a significant reduction in the luminosity, the crossing angle $\theta$ needs to be smaller than $\theta_{0}$.

There are several schemes for crabbing of the electron beam. One way is to use transversely deflecting cavities [1], such as an RF cavity working in the transverse mode $\mathrm{TEM}_{110}$. Such a scheme is schematically shown in Fig.(3). Another scheme is called dispersive crabbing [5]. In this scheme, an RF cavity is used to introduce an energy chirping along the beam, and due to dispersion of the beam transport system the beam will be tilted in transverse angle.

\section{Crabbing of the Photon Beam}

The geometrical reduction in Compton scattering efficiency due to a crossing angle is given by the same equations, Eq.(2) and Eq.(3). For typical parameters for a $\gamma-\gamma$ collider, $\theta_{0}$ is of the order of $1 \mathrm{mrad}$ for $\gamma-\gamma$ collision, and $10 \mathrm{mrad}$ for Compton scattering. Since the crossing angle may need to be as large as $30 \mathrm{mrad}$ [4], it is desirable to crab the laser beam as well as the electron beam, as shown in Fig.(1).

The crabbing of the photon beam from a laser may also be done in a number of ways. One way that is analogous to the dispersive crabbing mentioned above for the electron beam is shown in Fig.(4). In this scheme, a chirping in frequency is produced along the bunch length. Then by passing the chirped pulse through a dispersive optical element, such as a grating pair, the bunch will be tilted as well as compressed longitudinally.

In the geometrical optics approximation, the crabbing angle from the arrangement in Fig.(4) is straightforward to work out. The grating should be blazed for high efficiency operation in first order, with the grating equation $\cos \alpha-\cos \beta=g \lambda$, where $\alpha$ and $\beta$ are respectively the grazing angle of the incident and outgoing beams, and $g$ is the number of grooves per unit length. Let $\ell$ be the initial laser pulse length, $d$ be the separation of the 
two gratings, and $\Delta \lambda=\lambda_{2}-\lambda_{1}$ be the wavelength variation across the pulse. The pulse, after the grating pair, is shortened by a length $(\Delta l)_{\mathrm{z}}=\mathrm{dg}^{2} \lambda \Delta \lambda / \sin ^{3} \beta$, and crabbed with a transverse dimension $(\Delta \ell)_{\mathrm{T}}=\mathrm{dg} \Delta \lambda \sin _{\alpha} / \sin ^{3} \beta$. Then the crabbing angle $\theta_{\mathrm{c}}$ is given by

$$
\tan \theta_{c}=\frac{(\Delta \ell)_{T}}{\ell-(\Delta \ell)_{z}}
$$

This expression is valid for the case $(\Delta \ell)_{\mathrm{T}}$ is larger than the transverse size of the laser pulse. As an example, consider the case $\lambda=1 \mu \mathrm{m}, \Delta \lambda / \lambda=1 \%, \mathrm{~g}=100$ grooves $/ \mathrm{mm}, \alpha=45^{\circ}, \beta=52.6^{\circ}, \mathrm{d}=1 \mathrm{~cm}, \ell=500 \mu \mathrm{m}$. We obtain $(\Delta \ell)_{\mathrm{T}}=14 \mu \mathrm{m},(\Delta \ell)_{z}$ $=2 \mu \mathrm{m}$. Thus we have $\theta_{c}=28 \mathrm{mrad}$, which is in the correct order of magnitude.

Conventional laser systems producing high power, short pulse beams contain chirping and dispersive elements. The crabbed laser beam can simply be obtained by adjustments of these elements. In the case of free-electron lasers, on the other hand, the crabbing can be done externally.

\section{Conclusions}

It is seen that crab crossings can be devised for a $\gamma-\gamma$ collider at both conversion and collision points without reduction in either conversion efficiency or luminosity, and with no more difficulty than for an electron-positron collider.

\section{References}

R. Palmer, SLAC-PUB-4707 (1988).

[2] K. Oide and K. Yokoya, Phys. Rev. A, 40 (1989) 315.

[3] V. I. Telnov, Nucl. Instr. and Meth., A294 (1990) 72.

[4] V. A. Alexandrov, E. A. Kushnirenko, A. A. Sery and N. A. Solyak, in these proceedings.

[5] G. Jackson, AIP Conf. Proc. 214 (1990) 327. 


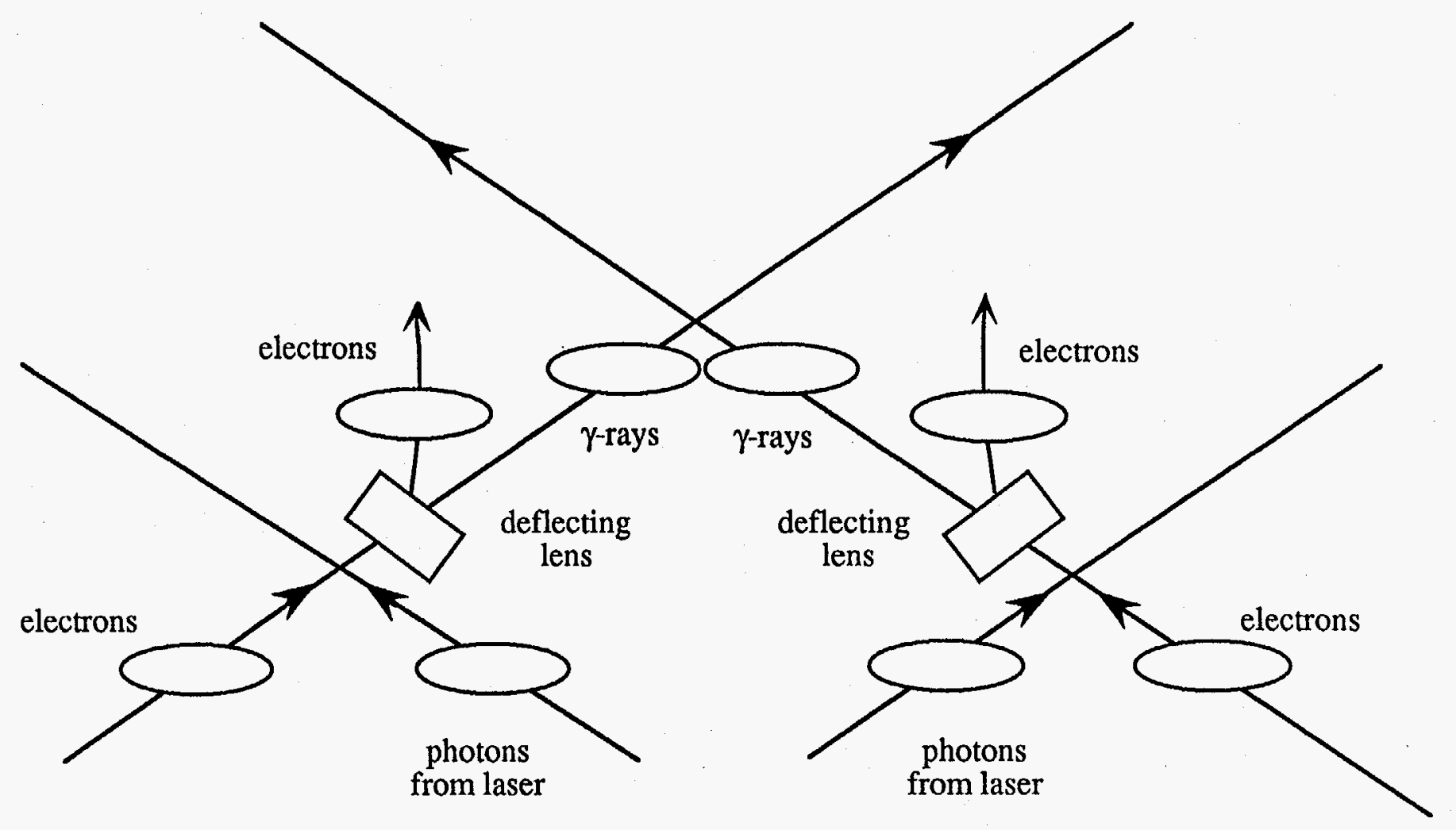

Fig. 1 Schematic of the conversion point and the collision point for a crabbed $\gamma-\gamma$ collider 


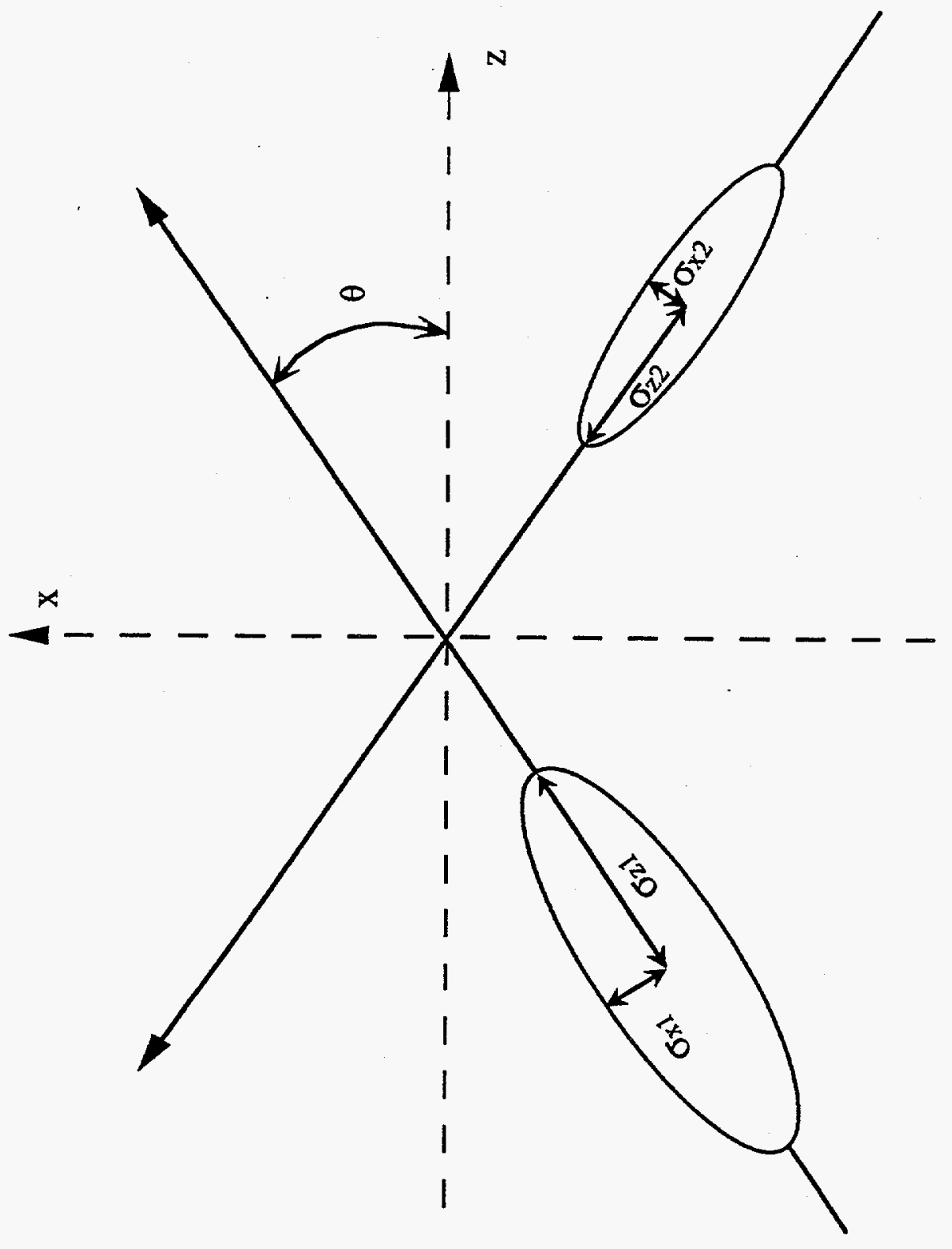

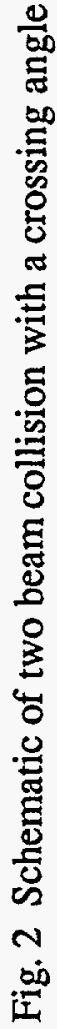




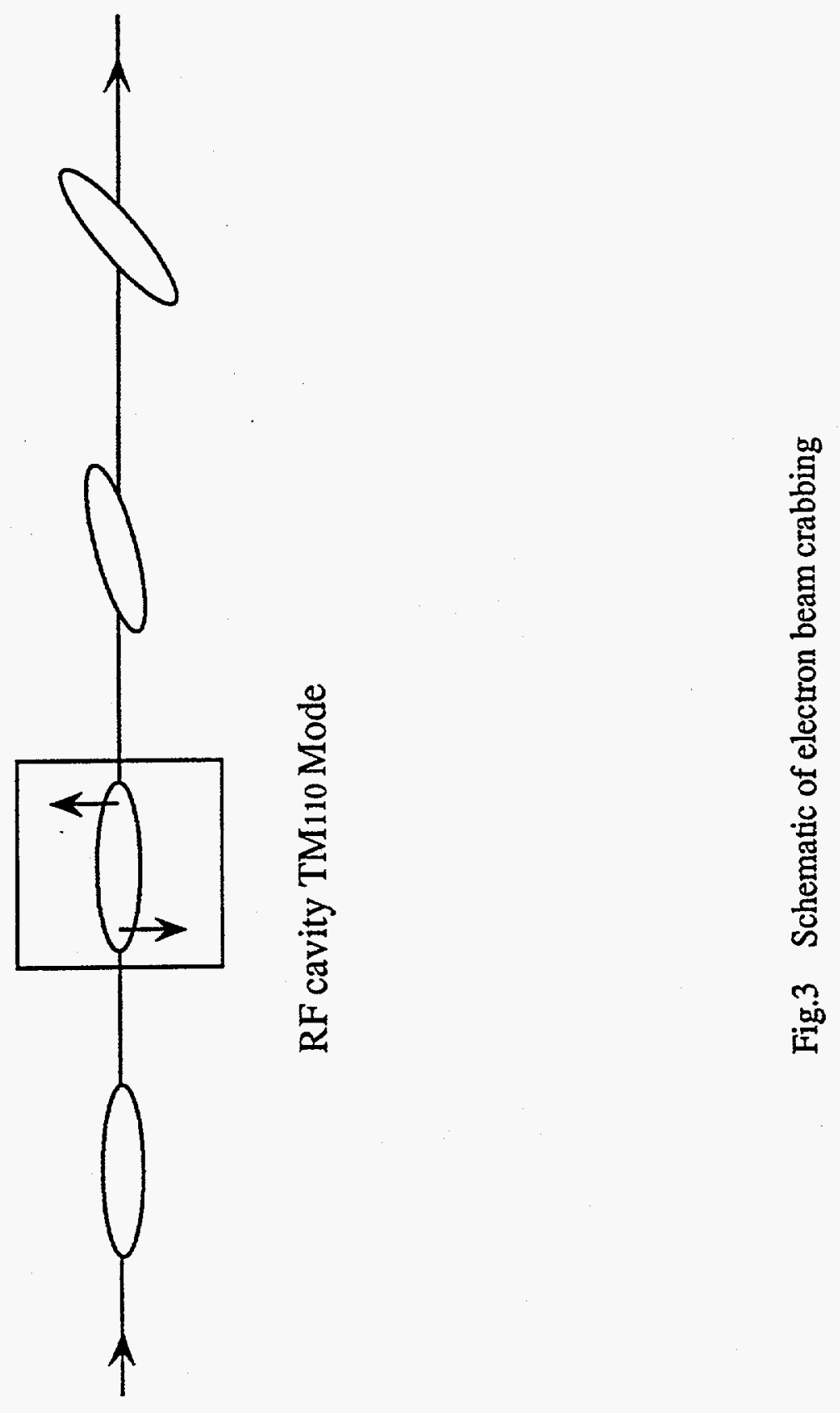




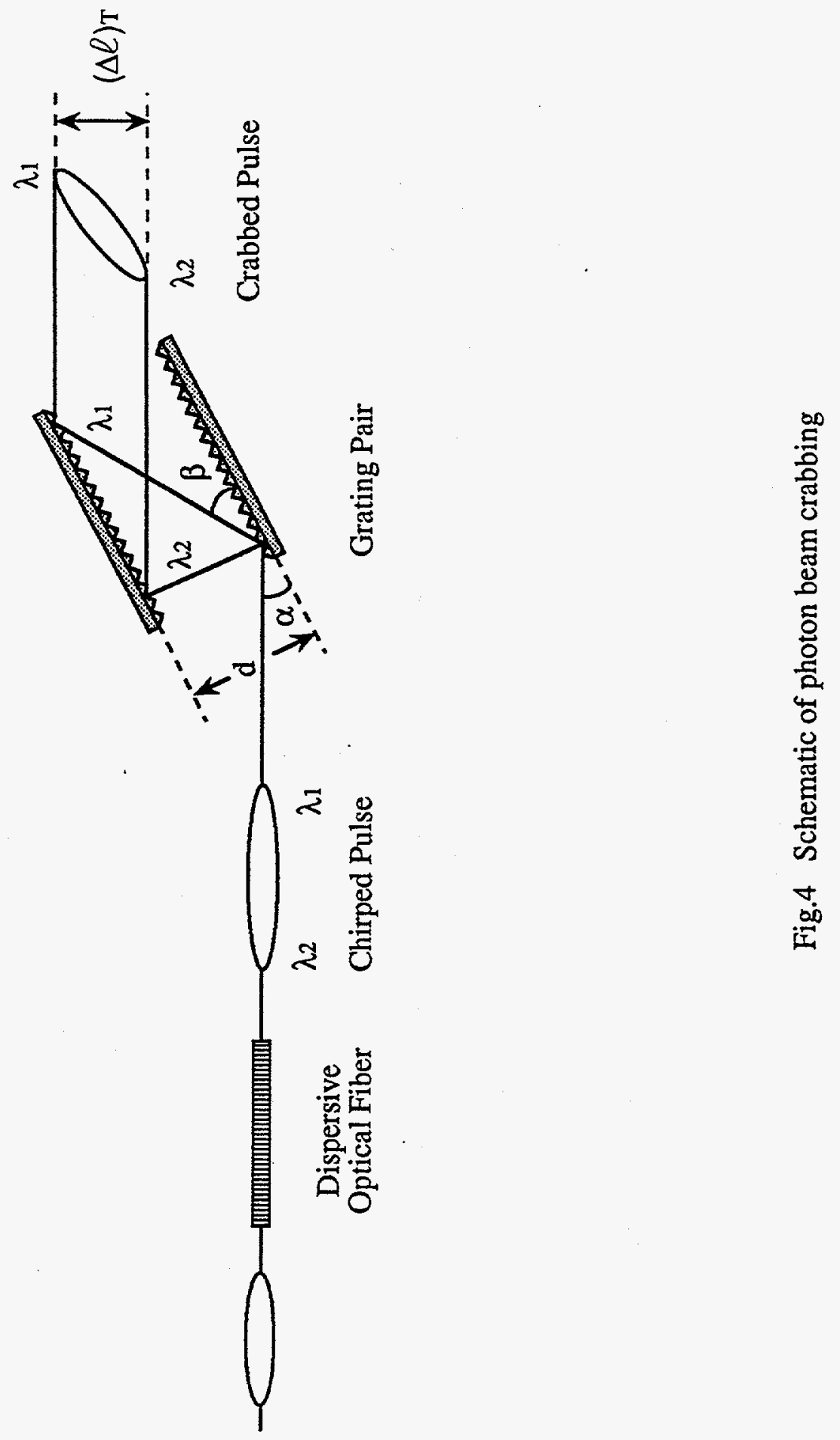

